weniger Unternehmen, und zweitens klare Analysen, welche Faktoren die ökonomische Entwicklung eines Unternehmen beeinflussen. Hier wird man dann feststellen, dass schon die Definition dessen, was ökonomischer Erfolg ist, nicht ganz einfach ist, und dass zudem so viele Faktoren darauf einwirken, ob zum Beispiel die Aktienkurse steigen oder fallen, dass es methodisch sehr ausgefeilter Analysen bedarf, um den Einfluss des Boards $\mathrm{zu}$ isolieren von all den anderen: Turbulenzen an den Börsen, generelle oder branchenspezifische Einbrüche in der Nachfrage, Fehler im Investitionsverhalten et cetera. Hier werden die Entwicklungen in Norwegen als dem Land mit der bisher radikalsten Veränderung sicherlich genau zu untersuchen sein und könnten in einigen Jahren tatsächlich Anhaltspunkte bieten für fundierte Analysen.

Es ist aus Sicht der Wissenschaft also schwierig, beim heutigen Zustand zu sagen: Mehr Frauen in Führungspositionen, in Vorständen und Aufsichtsräten führen zu besseren Renditen, mehr Gewinnen, einem besseren Arbeitsklima, et cetera. Die Forderung nach Gleichstellung, nach Zugang zu allen Positionen in Wirtschaft und Gesellschaft unabhängig vom Geschlecht ist aus meiner Sicht aber auch dann berechtigt, wenn mehr Frauen nicht unbedingt zu mehr Profit beitragen - oder will jemand ernsthaft behaupten, dass Gleichstellung nur dann zu fordern ist wenn es als „business case“ wirkt? Im Gegenteil: Aus meinen Erkenntnissen über das Funktionieren von Gleichstellung folgen folgende Empfehlungen, wie der Wandel gelingen kann:

1. Es geht um den gleichberechtigten Zugang von Frauen zu allen Positionen in Wirtschaft und Gesellschaft;

2. Die Unternehmen nicht nur in Deutschland sind von sich aus nur begrenzt in der Lage, diese Anforderung erfüllen zu können;

3. Deswegen bedarf es verbindlicher Regeln, die durch das Parlament beschlossen werden müssen, denn es handelt sich um die Realisierung elementarer Prinzipien unserer Demokratie;

4. diese verbindlichen Regeln müssen so definiert sein, dass keine kleinen Minderheiten entstehen, es muss gleich eine kritische Masse erreicht werden;

5. Das heißt ich spreche mich dafür aus, verbindliche Quoten festzulegen, die in einem definierten Zeitraum erreicht wer- den müssen; so, wie das einige europäische Länder, nicht nur Norwegen, schon beschlossen haben.

6. Die ökonomische Entwicklung der bundesdeutschen Unternehmen kann davon positiv beeinflusst werden; ob das passiert, das werden wir dann sehen. Ein unternehmerisches Scheitern wegen zu vieler Frauen „an Bord“ ist dagegen noch nicht berichtet worden. Auch der letzte große Insolvenzfall Schlecker hat mit den vielen Frauen in der Belegschaft jahrzehntelang erhebliche Gewinne gemacht - die Fehlentscheidungen haben nicht die Frauen zu verantworten, die Folgen dieser Fehlentscheidungen allerdings schon!

7. Unabhängig von einer gesetzlichen Quotenregelung wird es unumgänglich sein, die personalpolitischen Strategien in den Unternehmen zu verändern, denn nicht nur an der Spitze der Unternehmen steht Wandel an, sondern auch im Personalmanagement insgesamt: Eine größere Vielfalt in den Belegschaften und im Führungspersonal muss überlegt „gemanagt“ werden, denn Vielfalt hat viele positive Seiten, diese kommen aber nicht automatisch zum Tragen, sondern müssen aktiv befördert werden.

Deswegen ist mein Plädoyer, gemeinsam mit vielen anderen Wissenschaftler/inne/n:

- Gleichstellung und Vielfalt müssen in den Unternehmenszielen und Leitbildern verankert sein;

- Es müssen verbindliche Zeitpläne mit Verantwortlichkeiten und Anreizen beziehungsweise Sanktionen festgelegt werden;

- Die Transparenz bei der Besetzung von Führungspositionen, insbesondere in Top-Positionen, muss erhöht werden;

- Die Personalentwicklung muss so gestaltet werden, dass Frauen in die Karriereförderung einbezogen werden, flexiblere Karrieremöglichkeiten für Männer wie Frauen entwickelt werden und Familienarbeit für beide Geschlechter als ein positiver Faktor in der Personalentwicklung verankert wird;

- Man muss die herrschenden Geschlechterstereotype in den Unternehmenskulturen aktiv hinterfragen und damit offener und moderner auf die Lebenswirklichkeit der Menschen eingehen.

Nichts spricht dagegen, dass dies den Unternehmen, gleich welcher Branche, nutzen wird und alles spricht dafür, dass dies ein Beitrag zu mehr Geschlechtergerechtigkeit sein wird.

\title{
Wie kann die angemessene Teilhabe erreicht werden?
}

\author{
Monika Schulz-Strelow \\ Präsidentin von FidAR - Frauen in die Aufsichtsräte e.V., Berlin \\ Sehr geehrte Frau Pisal, \\ sehr geehrter Herr Botschafter, \\ diese Frage beschäftigt uns gemeinsam seit Jahren. Herz- \\ lichen Dank, dass wir hier im März, dem Monat der vielen
}

politischen Proklamationen am Internationalen Frauentag und notwendigen Aktionen zum Equal Pay Day, aber wenig sichtbaren Veränderungen, zusammenkommen und uns aus unterschiedlicher Sicht mit der aktuellen Situation von Frauen in Aufsichtsräten oder besser von fehlenden Frauen befassen.

Herr Botschafter, wir erinnern uns an das 1. FidAR-Forum im September 2009 in der britischen Botschaft, wo wir leicht 
euphorisch aber auch ungläubig den Stufenplan-Vorschlag für mehr Frauen in Aufsichtsräten durch den damaligen Staatssekretär des Familienministeriums erläutert bekamen. Was ist daraus geworden? Außer Ankündigungen einer „Flexiquote " bisher sehr wenig und meiner Meinung wird in dieser Legislaturperiode auch nicht mehr viel passieren.

Aufmerksam registrieren wir jede noch so marginale Veränderung, die das DIW jeweils im Januar mit dem „Managerinnenbarometer“ zu den 200 größten Unternehmen veröffentlicht. Fazit: es bewegt sich sehr wenig.

FidAR hat im letzten Jahr den „Women-on Board“-Index der Öffentlichkeit präsentiert. Mit dem „WoB-Index“ greifen wir die Empfehlungen der deutschen Corporate Governance Kodex Kommission aus dem Jahr 2010 auf, die Anzahl der Frauen im Aufsichtsrat und Vorstand der 160 börsennotierten DAX-, MDAX-, SDAX- und TecDAX-Unternehmen transparent zu machen und die Veränderungen zu dokumentieren. Die Veränderungen sind gering, der Anteil von Frauen ebenfalls:

- In den Aufsichtsräten und Vorständen sind nur 10,6 Prozent Frauen vertreten (von 2275 halten Frauen 216 Mandate);

- In den Aufsichtsräten sind es 11,8 Prozent Frauen (192 von 1621);

- Betrachtet man bei den Aufsichtsräten nur die Vertreterinnen der Kapitalseite, sind es nur 4,6 Prozent;

- Kleineren Unternehmen (SDAX, MDAX) gelingt es besser, das Potential von Frauen nutzen;

- 54 von 160 Unternehmen (37,5\%) sind im Aufsichtsrat und Vorstand frauenfrei!

Dieses vom Familienministerium geförderte Projekt und der in Zusammenarbeit mit dem manager magazin herausgebrachte WoB-Index erfährt höchste Aufmerksamkeit. Zumindest hat sich die „frauenfreie Zone“, das heißt die Unternehmen, die weder eine Frau im Aufsichtsrat noch im Vorstand haben, von 76 in 2011 auf 54 in 2012 verringert. Doch gibt unter den „Nullern“ Bollwerke mit 20-köpfigen Aufsichtsräten und sechsköpfigem Vorstand wie die Südzucker AG. Die ebenfalls frauenfreie Fresenius SE argumentiert, dass sie Positionen nur nach Qualität besetzt. Wir haben zum Jahreswechsel den Aufsichtsräten geschrieben und die jeweilige Veränderung, ob Verbesserung, gleichbleibende Position oder auch Verschlechterung der Position im WoB-Index, dokumentiert. Mehrere Aufsichtsräte haben geantwortet und ihre Pläne erläutert und teilweise wiederholt betont, dass nur nach Qualität ausgewählt wird. Fazit: Es gibt noch viel Raum für Veränderung.

Wenn wir nur 1,5 Prozent Erhöhung bei den Vorstandsbesetzungen feststellen können und Studien belegen, man braucht so viel Zeit zur Umsetzung, wie man geplant hat, kann sich jeder hier im Saal ausrechnen, was für eine lange Durststrecke noch vor uns liegt. Und wenn BMW im Oktober 2011 im Rahmen der „DAX-30-Erklärung“ als Ziel definiert, 15 Prozent Frauen in Führungspositionen im Jahr $2020 \mathrm{zu}$ erreichen, dann werden sie auch kaum bei einer höheren Prozentzahl im Jahr 2020 liegen. Allerdings bei Modellentwicklungen und Positionierung im Konzert der Automobilindustrie sind sie wesentlich ambitionierter und zeitnaher. Wenn allerdings diese marginale 1,5-ProzentVerbesserung im WoB-Index einem Koalitionspartner in der Bundesregierung als Nachweis ausreicht, dass wir keine Quote oder andere Instrumente brauchen, ehrt es uns einerseits, dass dem WoB-Index so viel Aufmerksamkeit zuteil wird. Andererseits ist es schon sehr erstaunlich, dass ein Ergebnis so missinterpretiert werden kann und dies hinterlässt einen bitteren Nachgeschmack. Da aber nicht nur Männer Zahlen und Rankings verstehen, sondern auch Frauen gut zählen und wählen können, erwarte ich Reaktionen bei den anstehenden Wahlen.

Ähnlich überrascht war ich vorletzte Woche, als ich in einem Interview eine Äußerung der Familienministerin zu der „Pro-Quote“-Aktion der Journalistinnen las, dass diese Aktion zeige, dass wir keine gesetzliche Quote und auch keine Flexiquote brauchen, da Branchen dies intern regeln werden. Vielleicht verstehe ich die Forderung der Journalistinnen nicht richtig. Da auf gesetzlicher Ebene nichts passiert und der Zustand in ihrer Branche sich nicht verbessert, haben sie die Form dieses Aufrufs mit einem 30-Prozent-Frauenanteil auf allen Hierachieebenen in der Medienbranche gewählt und entsprechende Aufmerksamkeit gefunden. Es arbeiten zwar viele Frauen in den Medien; doch die Meinungsmacher sind weiterhin die Männer.

Es heißt, es bewegt sich einiges, stimmt. Dazu haben wir alle hier im Raum an unterschiedlichen Stellschrauben wesentlich beigetragen und häufig nicht nur freundliche Blicke und Worte geerntet, wie die djb-Vertreterinnen auf den Hauptversammlungen bestimmt auch berichten können. Doch wir haben nur durch diese sich ergänzenden Aktionen und Instrumente in so kurzer Zeit eine so weitreichende Wirkung in den Medien, bei Unternehmen und bei der deutschen Corporate Governance Kodex-Kommission erzielen können. Auch die Politik reagiert, aber bisher nur sehr schleppend.

An dieser Stelle möchte ich auch auf die Initiative der „Berliner Erklärung“ Bezug nehmen. Die Forderung nach „30 Prozent“ in den Aufsichtsräten teilen alle Initiatorinnen der Frauenverbände und die Repräsentantinnen aller im Parlament vertretenen Parteien. Das Ziel der Abgeordneten des Bundestags ist, einen gemeinsamen Antrag für mehr Frauen in Aufsichtsräte einzureichen. Dieses Ziel unterstützen wir mit dem Einsammeln von Unterschriften, um dem Anliegen mehr Öffentlichkeit zu verschaffen und damit zu verdeutlichen, dass das Thema in der breiten Öffentlichkeit auch angekommen ist. Wir wollen erreichen, dass im Parlament fraktionsübergreifend an zentralen Themen gearbeitet wird und nicht an den Fraktionsgrenzen Halt gemacht wird. Das verstehen die Menschen draußen nicht mehr und die Abgrenzungen tun dem Anliegen nicht gut. Doch mit der „Berliner Erklärung " machen wir auch deutlich, dass die gemeinsamen Forderungen eine gesellschaftliche Veränderung und anderen Umgang miteinander erfordern. 
Das Thema „Frauen in Führung“ sollte noch viel breiter diskutiert werden, damit es nicht auf ein Luxusproblem reduziert wird. Durch unsere bisherige Arbeit hat es meiner Meinung nach in den Unternehmen, die unserer Forderung mit folgenden Argumenten lange ausweichend begegnen wollten, wie, man müsse von unten die jungen Mädchen schon in der Schule anders fördern, auch ein Umdenken gegeben. Unternehmen kommen an dem Thema Frauenförderung nicht mehr vorbei und gehen es teilweise auch schon ganzheitlich an. Die klassische Frauenförderung mit wenig messbaren Ergebnissen wird häufig durch innovative Ansätze abgelöst. Diese neue Denkweise wird sich in den Unternehmen positiv niederschlagen, die das Thema ernst nehmen. Dies erkennt man daran, ob das Thema als Teil der Unternehmensstrategie beim CEO angesiedelt ist. Das wäre zumindest die richtige Ebene. Es ist zu beobachten, dass die Sprache und Kommunikationskultur sich verändert, wenn das Thema zentrale Bedeutung erfährt. Dafür ist die Sprache des CEOs ein sicherer Prüfstein. Nichts ist heute gefährlicher als dahingeworfene Sätze wie „bunt und schön“, die in Sekundenschnelle per Twitter weltweit verbreitet werden und eine nicht beabsichtigte Wirkung erzielen.

Die Unternehmen können daran gemessen werden, welche Schritte sie ergreifen und wie sie im internationalen Vergleich stehen. Sich wegen Fachkräftemangel und demographischem Wandel und schwellendem politischen Druck mit Frauenförderung zu befassen, wird nicht ausreichen. Für die zukunftsweisenden Unternehmensführungen werden die Führungsmannschaften eine Veränderung durchlaufen müssen, da ein anderes Verständnis für gemischte Gremien gefordert ist, damit es nachhaltig funktioniert. Außerdem sind Programme notwendig, die Frauen nicht nur fördern, sondern befördern! Die Ergebnisse müssen überprüfbar sein, und als wirksame Maßnahme am jeweiligen Bonus festgemacht, werden die Ergebnisse auch anders ausfallen. Diese Koppelung beschleunigt ungemein.

Hier sind auch die Frauen in Führungspositionen gefragt, die ihre Vorbildfunktion anders wahrnehmen müssten und nicht als erstes öffentlich erklären, sie haben alles nur aus eigener Leistung geschafft und möchten mit dem Frauenthema nicht in Verbindung gebracht werden. Alleine den Weg nach oben schafft keiner und keine - da ist es auch völlig legitim sich der Netzwerke zu bedienen. Der Zugang zu den richtigen Netzwerken spielt dabei eine wichtige Rolle. Hier sind Frauen mit Durchhaltevermögen gefragt, die nach oben wollen und nicht bei dem Wort „Macht“ einen Rückzieher machen. Auch sind die jungen, hochqualifizierten Frauen gefragt, die ihren Karriereweg viel klarer definieren müssen als es die „glass-ceiling-Fraktionen “ wahrscheinlich weitgehend gemacht haben.

Und hier sind Männer gefragt, für die eine andere Unternehmenskultur mit weniger Präsenzanforderungen erstrebenswert ist, die andere Lebensmuster für sich anstreben und die die Vereinbarkeit von Familie und Karriere auch als ihr Thema ansehen.

Leider wird der Weg dahin, zum Überwinden der bestehenden Barrieren, weiterhin steinig und wenig gemütlich sein - wir befinden uns nicht in der Komfortzone. Doch fehlen uns zum Beschleunigen des Weges die richtigen Instrumente. Da hilft nur ein richtiger Schub, um unser Ziel kurz- bis mittelfristig zu erreichen. Ich sehe die verbindliche Quote für die Aufsichtsräte als das ressourcenschonendste und wirksamste Instrument an. Wir brauchen sie für den Anschub und als Wegbeschleuniger, leider auch mit gesetzlicher Grundlage - alles andere wirkt in Deutschland nicht so nachhaltig. Wie sagte die EU-Kommissarin Viviane Reding unlängst in Berlin so treffend: „Ich liebe nicht die Quote - aber ihre Ergebnisse“. Diese Meinung teile ich uneingeschränkt.

Meine Bitte an uns alle: Gehen wir die gleiche Teilhabe von Frauen und Männern mit unterschiedlichen Instrumenten an. Wir haben mit der djb-Hauptversammlungsaktion, dem VdU-Seminar und der Datenbank-Initiative, mit dem „Equal-Pay-Day“ vom BPW, der „Managerinnen50+“Studie des EWMD und dem WoB-Index von FidAR eine Reihe von sich ergänzenden Instrumenten entwickelt sowie mit der Berliner Erklärung eine gemeinsame Initiative mit Vertreterinnen von allen im Bundestag vertretenen Parteien ins Leben gerufen, die gebündelt mit entsprechendem Nachdruck unser Ziel verstärken. Die zentralen Frauenverbände arbeiten nicht nebeneinander, sondern miteinander, um das gemeinsame Ziel zu erreichen. Ich glaube, dieser Ansatz ist eines der wertvollsten Ergebnisse der letzten fünf Jahre: dass unabhängige Frauenverbände miteinander gemeinsam ein Anliegen nach vorne bewegen. Und dafür lohnt es, sich auch weiterhin einzusetzen.

Doch als Wichtigstes brauchen wir begeisterungsfähige Menschen, die unser Land gleichberechtigt nach vorne bringen. Dann würden viele der laufenden Diskussionen überflüssig sein und wir könnten uns der inhaltlichen Arbeit und Umsetzung zuwenden, die eigentlich auf uns wartet.

Herzlichen Dank für Ihre Aufmerksamkeit. Ich hoffe auf eine große Anzahl von Unterschriften auf den Berliner Erklärungslisten. 